\title{
ORBITAL STRUCTURE OF TRIAXIAL EQUILIBRIUM MODELS OF VARIOUS SHAPES
}

\author{
S. UDRY and L. MARTINET \\ Geneva Observatory, CH-1290 Sauverny, Switzerland
}

\section{Aim}

To bring out the link between the observed shapes of triaxial dynamical systems (ellipticals, bulges, bars) and their orbital populations inferred from models.

\section{Methods}

1) We consider a series of triaxial systems of various shapes (elliptic, boxy, peanut, disky) built from N-body simulations of collisionless gravitational collapses. The used code is characterized by: a particle mesh algorithm; a polar grid exponentially spaced in the radial direction insuring a good central resolution; 200000 particles; for details see Udry 1992 and Pfenniger \& Friedli 1992.

2) We randomly select in each $\mathrm{N}$-body configuration a set of about 2000 particles and integrate the motion over more than $5 \cdot 10^{9} \mathrm{y}$ in the fixed stabilized potentials.

3) We define parameters $n_{i}$ as the numbers of sign changes of any coordinate $i\left(i=x, y\right.$ or $z$ ) along the orbit. We build histograms of $n_{i} / n_{j}$, normalized to the number of considered particles, and corresponding to the ratio $\omega_{i} / \omega_{j}$ of orbital oscillation frequencies along the $i$ - and $j$-axes.

4) We estimate the percentage of orbits with frequency ratios in various intervals corresponding to tube-, round-, boxy- or peanut-shaped orbits. The more populated intervals of frequency ratios and consequently the corresponding orbits may be related to the global shape of the models.

5) To establish a shape - orbital population relation, we calculate for each model the normalized fourth coefficient of Fourier analysis of the isophote departures from pure ellipses, $a_{4} / a$, which gives the degree of boxiness of the isodensity contours (Bender \& Möllenhoff 1987). It is also compared to the model flattenings.

6) By means of Floquet theory, we have estimated $\omega_{z} / \omega_{x}$, the ratio of the normal frequency in the $z$ direction to the orbital frequency of the $x$-axial orbit, in a peanut-shaped model and a boxy shaped model.

\section{Results}

1) Their is a clear correlation between the model shapes and their orbital populations parametrized by oscillation frequency ratios along the figure's main axes $\left(n_{i} / n_{j}\right.$; Figure 1$)$. Peanut-shaped models present a significant proportion of orbits with $n_{i} / n_{j}>3 / 2$, whereas orbits in elliptical-shaped systems are rather characterized by $1 \lesssim n_{i} / n_{j} \lesssim 3 / 2$. This result implies a relation between Bender's 


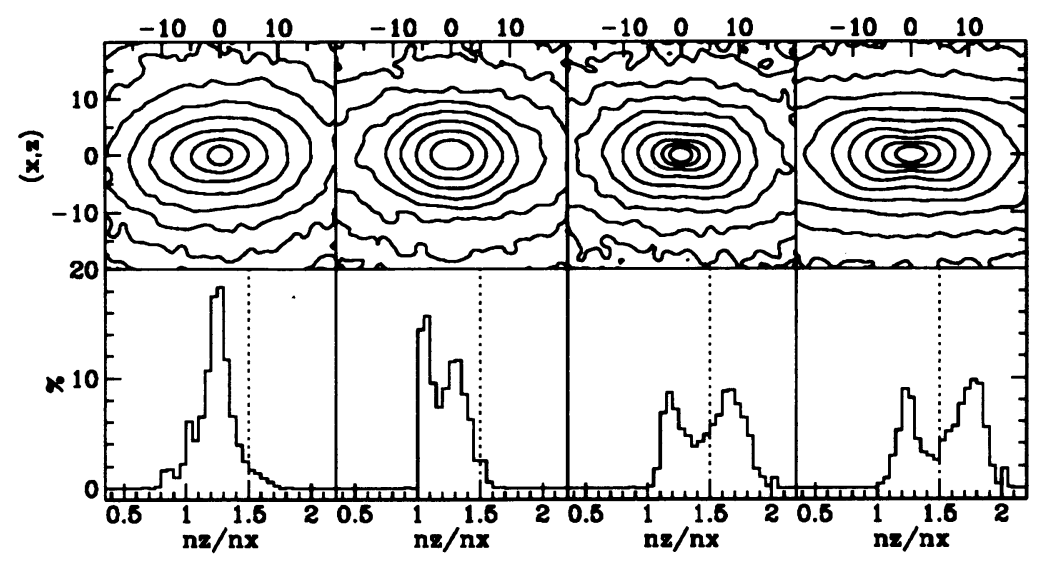

Fig. 1. Projected density contours in the $(x, z)$ plane for an elliptic-boxy-peanut sequence of obtained N-body equilibrium models with corresponding normalized histograms of $n_{z} / n_{x}$ values. Parameters $n_{i}$ are the numbers of sign changes of the coordinate $i(i=x$ or $z)$ along the orbit $\left(n_{z} / n_{x} \simeq \omega_{z} / \omega_{x}\right)$.

$a_{4} / a$ as a shape parameter and the percentage of orbits whose $n_{i} / n_{j}$ values are in a particular interval, as an orbital population parameter.

2) We also observe a well defined relation between the degree of boxiness of the models and the configuration flattenings in the sense that the more flattened a model is, the more boxy- or peanut-shaped it looks like. This relation is due to the individual shape of resonant orbits whose vertical oscillation frequencies increase $\left(n_{z} / n_{x}\right.$ goes up) as their $z$-amplitudes go down.

3) The global model configuration depends on the sum of the effective populations of the existing orbital classes, and thus is related to the characteristics of the more numerous ones. In models with the particular oblate or prolate symmetries, the circular shape is induced by tube orbits in the corresponding planes. On the contrary, in triaxial models, tube orbits are not numerous and round shapes are rather given by resonant orbits with low $n_{i} / n_{j}(\sim 1)$.

4) The approximate percentage of box orbits is between 60 and $70 \%$ in strongly triaxial systems and less than $30 \%$ in prolate or oblate non rotating ones.

5 ) Finally, the vertical instability on the $x$-axial orbit, induced by the bifurcation with vertical banana orbit families in boxy- and peanut-shaped models, contributes to push motions out from the equatorial plane and thus to populate orbits determining the global shape of the system.

Details will be published elsewhere (Astronomy \& Astrophysics).

\section{References}

Bender R., Möllenhoff C., 1987, A\&A, 177, 71

Pfenniger D., Friedli D., 1992, submitted

Udry S., 1992, A\&A, in press 